TRANSACTIONS OF THE

AMERICAN MATHEMATICAL SOCIETY

Volume 364, Number 10, October 2012, Pages 5149-5172

S 0002-9947(2012)05447-X

Article electronically published on May 7, 2012

\title{
FRACTIONAL DERIVATIVE OF ABEL TYPE ON A HALF-LINE
}

\section{ELENA I. KAIKINA}

Abstract. We consider the initial-boundary value problem on a half-line for an evolution equation

$$
\left(\partial_{t}+\left|\partial_{x}\right|^{\alpha}\right) u(x, t)=f(x, t), t>0, x>0,
$$

with a fractional derivative of Abel type

$$
\left|\partial_{x}\right|^{\alpha} u=\mathcal{R}^{1-\alpha+[\alpha]} \partial_{x}^{[\alpha]+1} u
$$

where $[\alpha]$ denotes the integer part of number $\alpha>0, \alpha$ is not equal to an integer, and

$$
\mathcal{R}^{\alpha} u=\frac{1}{2 \Gamma(\alpha) \sin \left(\frac{\pi}{2} \alpha\right)} \int_{0}^{+\infty} \frac{\operatorname{sign}(x-y) u(y)}{|x-y|^{1-\alpha}} d y
$$

is the modified Riesz potential. We study traditionally important problems of a theory of partial differential equations, such as existence and uniqueness of solution. We propose a new method of solution. Also we get a closed form of the solution.

\section{INTRODUCTION}

In this paper we study the initial-boundary value problem on a half-line for an evolution equation with a fractional derivative of Abel type,

$$
\left\{\begin{array}{c}
\left(\partial_{t}+\left|\partial_{x}\right|^{\alpha}\right) u(x, t)=f(x, t), t>0, x>0, \\
u(x, 0)=u_{0}(x), x>0, \\
\partial_{x}^{j} u(0, t)=h_{j}(t), t>0, j=0, \ldots,[\alpha]+1-\left[\frac{\alpha+1}{2}\right],
\end{array}\right.
$$

where $\alpha>0$ is not equal to an integer and the fractional derivative of Abel type $\left|\partial_{x}\right|^{\alpha}$ on a half-line is defined as

$$
\left|\partial_{x}\right|^{\alpha} u=\mathcal{R}^{1-\alpha+[\alpha]} \partial_{x}^{[\alpha]+1} u,
$$

where $[\alpha]$ denotes the integer part of number $\alpha>0$ and

$$
\mathcal{R}^{\alpha} u=\frac{1}{2 \Gamma(\alpha) \sin \left(\frac{\pi}{2} \alpha\right)} \int_{0}^{+\infty} \frac{\operatorname{sign}(x-y) u(y)}{|x-y|^{1-\alpha}} d y
$$

is the modified Riesz potential (see [14], p. 214). Note that the main basic properties of the modified Riesz potential, such as the mapping properties, inverse operator, index rule, etc., were studied in [14]. Due to the intensive development of the theory of fractional calculus itself as well as its applications ([7], [8], [14]), the initial-boundary value problem (1.1) plays an important role in the modern science. Apart from diverse areas of mathematics, evolution partial differential equations

Received by the editors June 11, 2010 and, in revised form, August 20, 2010.

2010 Mathematics Subject Classification. Primary 35Q35.

Key words and phrases. Initial-boundary value problem, Green function, fractional derivative.

(C)2012 American Mathematical Society Reverts to public domain 28 years from publication 
with a fractional derivative arise in modern mathematical physics and many other branches of science, such as, for example, chemical physics and electrical networks (for details, see, for example, [1, [2], 9], 10, 13], [15], 16]).

There are several definitions of a fractional derivative of order $a>0$ (for details, see, for example, [7, 8, 14]). The two most commonly used definitions are the Riemann-Liouville and Caputo ([14]). Each definition uses Riemann-Liouville fractional integration and derivatives of an integer order. The Riemann-Liouville fractional derivative of order $\alpha>0$ is defined as

$$
D^{\alpha} u=\frac{1}{\Gamma(1-\alpha+[\alpha])} \partial_{x}^{[\alpha]+1} \int_{0}^{x} \frac{u(y)}{(x-y)^{\alpha-[\alpha]}} d y, x>0,
$$

and the Caputo derivative of order $a>0$ is defined as

$$
D_{*}^{\alpha} u=\frac{1}{\Gamma(1-\alpha+[\alpha])} \int_{0}^{x} \frac{\partial_{y}^{[\alpha]+1} u(y)}{(x-y)^{\alpha-[\alpha]}} d y, x>0 .
$$

There is a close connection between the Riemann-Liouville fractional derivative and Caputo derivative (see [14]). To reveal a relation between the fractional derivative of Abel type and the Riemann-Liouville and Caputo fractional derivatives, we rewrite all of them as pseudo-differential operators on a half-line (see [4]). For simplicity we consider the case of $\alpha \in(0,1)$. Then we have

$$
\begin{gathered}
D^{\alpha} f(x)=\mathcal{L}^{-1}\left(p^{\alpha} \widehat{f}(p)\right), \\
D_{*}^{\alpha} f(x)=\mathcal{L}^{-1}\left(p^{\alpha}\left(\widehat{f}(p)-\frac{f(0)}{p}\right)\right)
\end{gathered}
$$

and

$$
\left|\partial_{x}\right|^{\alpha} u=\theta(x) \mathcal{L}^{-1}\left(|p|^{\alpha}\left(\widehat{f}(p)-\frac{f(0)}{p}\right)\right),
$$

where $\mathcal{L}^{-1}$ denotes the inverse Laplace transform and $\widehat{f}(p)=\mathcal{L} f$ is the Laplace transform of $f$. Here and below $p^{\alpha}$ is the main branch of the complex analytic function in the complex half-plane $\operatorname{Re} p \geq 0$, so that $1^{\alpha}=1$ (we make a cut along the negative real axis $(-\infty, 0))$, and the Heaviside step function $\theta(x)=0$ for $x<0$ and $\theta(x)=1$ for $x \geq 0$. As we see, the Riemann-Liouville and Caputo fractional derivatives have a symbol $p^{\alpha}$ which is analytic in the complex half-plane $\operatorname{Re} p \geq 0$. On the contrary the fractional derivative of Abel type is represented as a pseudo-differential operator with a nonanalytic symbol $|p|^{\alpha}$. Exactly this type of a nonanalytic symbol has appeared recently in different physical models for a better description of considered material properties. For example, the nonlocality of the dynamics of the ion-acoustic waves in plasma with Landau damping was modeled by the fractional derivative of Abel type. In papers [1], 12, the following equations were derived for the case of the real line $x \in \mathbf{R}$ :

$$
\begin{gathered}
u_{t}+u u_{x}+u+\alpha u_{x x x}+\int_{-\infty}^{+\infty} \frac{\operatorname{sign}(x-y) u_{y}(y, t)}{\sqrt{|x-y|}} d y=0, \\
u_{t}+u u_{x}+\alpha u_{x x x}-\int_{-\infty}^{+\infty} \frac{\operatorname{sign}(x-y) u_{y y}(y, t)}{\sqrt{|x-y|}} d y=0 .
\end{gathered}
$$

If we are interested in the case of a half-line $x>0$ (i.e., the function $u \equiv 0$ for all $x<0$ ), then we can see that the nonlocal terms of these equations convert to the fractional derivatives of Abel type of order $\alpha=\frac{1}{2}, \alpha=\frac{3}{2}$. 
Thus the same physical problems very often lead us naturally to an evolution partial differential equation with the fractional derivative of Abel type. So it is necessary to formulate correctly the initial-boundary value problem to such types of equations. It should be noted that most papers and books on fractional calculus are devoted to solvability of fractional ordinary differential equations (ODE). However, as far as we know, only a few works have considered the initial-boundary value problems for evolution partial differential equations with fractional derivatives not only of the Abel type but also classical fractional derivatives.

For the general theory of nonlinear equations on a half-line, we refer to the book [4. This book is the first attempt to develop systematically a general theory of the initial-boundary value problem for evolution equations with pseudo-differential operators on a half-line, where pseudo-differential operator $\mathbb{K}$ on a half-line was introduced by virtue of the inverse Laplace transformation of the product of the symbol $K(p)=O\left(p^{\alpha}\right)$ which is analytic in the right complex half-plane, and the Laplace transform of the derivative $\partial_{x}^{[\alpha]} u$. Thus, for example, in the case of $K(p)=$ $p^{\alpha}$, we get the following definition of the fractional derivative $\partial_{x}^{\alpha}$ :

$$
\partial_{x}^{\alpha}=\mathcal{L}^{-1}\left\{p^{\alpha}\left(\mathcal{L}-\sum_{j=1}^{[\alpha]} \frac{\lim _{x \rightarrow 0^{+}} \partial_{x}^{j-1}}{p^{j}}\right)\right\} .
$$

Note that due to the analyticity of $p^{\alpha}$ for all $\operatorname{Re} p>0$ the inverse Laplace transform gives us the function which is equal to 0 for all $x<0$. To obtain an explicit form of the Green function, an approach was used based on the Laplace transformation with respect to the spatial variable contrary to the standard application of the Laplace transformation with respect to the time variable. It was proved that the amount of boundary data which we need to put in the problem for its well posedness is equal to the integer part of $\left[\frac{\alpha}{2}\right]$, where $\alpha$ is the order of the operator $\mathbb{K}$, which is not equal to an odd integer (in the case of odd integer order of operator $\mathbb{K}$, the amount of the boundary data also depends on the sign of the highest derivative). Methods of this paper can be applied directly to study the initial-boundary value problem for differential equations with Caputo fractional derivative

$$
\partial_{x}^{\alpha}=\frac{1}{\Gamma(1-\alpha+[\alpha])} \int_{0}^{x} \frac{\partial_{y}^{[\alpha]+1}}{(x-y)^{\alpha-[\alpha]}} d y .
$$

Despite the importance and actuality there are few results about the initial-boundary value problem for pseudo-differential equations with nonanalytic symbols. For example, in paper [5] we considered the case of rational symbol $K(p)$ which have some poles in the right complex half-plane. A new method was proposed for constructing the Green operator based on the introduction of some necessary condition at the singularity points of the symbol $K(p)$. In the paper [6] we considered the initial-boundary value problem for a pseudo-differential equation with symbol $K(p)=|p|^{\frac{1}{2}}$. As far as we know, the case of general nonanalytic symbols $K(p)$ was not studied previously. In the present paper we fill this gap, considering an example of evolution equation with fractional derivative of Abel type (1.2) with a symbol $K(p)=|p|^{\alpha}$. There are many natural open questions which should be studied. First we need to consider the question, How many boundary data should be posed in problem (1.1) for its correct solvability? Also we study traditionally important problems of a theory of partial differential equations, such as existence and uniqueness of solution. Also we get the closed form of solution. 
The main difficulty for equation (1.1) on a half-line is that the symbol $K(p)=|p|^{\alpha}$ is nonanalytic in the complex plane. Therefore we cannot apply the Laplace theory directly. To construct a Green operator, we propose a new method based on the integral representation for the sectionally analytic function and theory of singular integro-differential equations with a Hilbert kernel and discontinuous coefficients. We prove that the amount of the boundary data which we need to put in the problem for its well posedness is equal to $[\alpha]+1-\left[\frac{\alpha+1}{2}\right]$, where $\alpha$ is the order of the operator $\left|\partial_{x}\right|^{\alpha}$, which is not equal to an integer. As we see from (1.2), the boundary values $\partial_{x}^{j-1} u(0, t), j=1, \ldots,[\alpha]+1$ are involved in the definition of $\left|\partial_{x}\right|^{\alpha}$; hence, the remainder $\left[\frac{\alpha+1}{2}\right]$ boundary values should be obtained as some necessary conditions for existence of solutions of the corresponding Riemann-Hilbert boundary value problem.

We believe that the results of this paper could be applicable in studying a wide class of dissipative nonlinear nonlocal equations on the half-line by using techniques of nonlinear analysis (estimations of Green function, fixed point theorems, etc., see [3]).

To state precisely the results of the present paper we give some notation.

Let $\mathbf{B}$ be a Banach space; we then denote

$$
\begin{aligned}
& \mathbf{C}^{k}([0, T], \mathbf{B}) \\
& =\left\{f(t) \in \mathbf{B}: \lim _{t_{1} \rightarrow t, t_{1} \in[0, T]}\left\|\partial_{t}^{k} f\left(t_{1}\right)-\partial_{t}^{k} f(t)\right\|_{\mathbf{B}}=0, \forall t \in[0, T]\right\} .
\end{aligned}
$$

Now we define the well posedness of the problem (1.1).

Definition 1. Problem (1.1) is called well posed in a semiclassical sense if the following two properties are fulfilled. First, there must exist a unique solution $u(x, t)$ belonging to a metric space

$$
\mathbf{C}^{0}\left([0, T], \mathbf{M}_{1}\right) \cap \mathbf{C}^{1}\left((0, T], \mathbf{M}_{2}\right),
$$

which satisfies equation $u_{t}+\left|\partial_{x}\right|^{\alpha} u=f$ in the generalized sense. Boundary and initial conditions are fulfilled in the classical sense

$$
\begin{aligned}
\lim _{t \rightarrow 0} u(x, t) & =u_{0}(x) \text { in } \mathbf{M}_{1} \text { and } \\
\lim _{x \rightarrow 0} \partial_{x}^{j-1} u(x, t) & =h_{j}(t) \text { in } \mathbf{C}^{0}([0, T]) \text { for all } j=1,2, \ldots, N .
\end{aligned}
$$

Second, solution $u(x, t)$ must be stable with respect to the initial data $u_{0}(x)$, boundary data $h_{j}(t)$ and a source $f(x, t)$. We call the function $u(x, t)$ a semiclassical solution. If $T=+\infty$, the function $u(x, t)$ is called a global semiclassical solution.

We denote $\langle t\rangle=1+t,\{t\}=\frac{t}{\langle t\rangle}$. The direct Laplace transformation $\mathcal{L}_{x \rightarrow \xi}$ is

$$
\widehat{u}(\xi) \equiv \mathcal{L}_{x \rightarrow \xi} u=\int_{0}^{+\infty} e^{-\xi x} u(x) d x,
$$

and the inverse Laplace transformation $\mathcal{L}_{\xi \rightarrow x}^{-1}$ is defined by

$$
u(x) \equiv \mathcal{L}_{\xi \rightarrow x}^{-1} \widehat{u}=(2 \pi i)^{-1} \int_{-i \infty}^{i \infty} e^{\xi x} u(\xi) d \xi
$$


The Lebesgue space is $\mathbf{L}^{q}\left(\mathbf{R}^{+}\right)=\left\{\varphi \in \mathcal{S}^{\prime} ;\|\varphi\|_{\mathbf{L}^{q}}<\infty,\right\}$ where

$$
\|\varphi\|_{\mathbf{L}^{q}}=\left(\int_{0}^{+\infty}|\varphi(x)|^{q} d x\right)^{\frac{1}{q}}
$$

for $1 \leq q<\infty$.

The Sobolev space is $\mathbf{H}_{2}^{n}\left(\mathbf{R}^{+}\right)=\left\{\varphi \in \mathbf{L}^{2} ; \sum_{j=0}^{n}\left\|\partial_{x}^{j} \varphi\right\|_{\mathbf{L}^{2}}<\infty\right\}$.

We denote

$$
K(q)=|q|^{\alpha}, K_{1}(q)=e^{i \pi\left[\frac{\alpha+1}{2}\right]} q^{\alpha} .
$$

We define

$$
\mathbb{G}(t) \phi=\int_{0}^{+\infty} G(x, y, t) \phi(y) d y,
$$

where for $x>0, y>0, t>0$ the function $G(x, y, t)$ is given by formula

$$
\begin{aligned}
G(x, y, t) & =\frac{1}{2 \pi i} \int_{-i \infty}^{i \infty} d p e^{p(x-y)} \frac{1}{K(p)+\xi} \\
& -\frac{1}{2 \pi i} \frac{1}{2 \pi i} \sum_{j=1}^{M} \int_{-i \infty}^{i \infty} d \xi e^{\xi t} \xi^{\frac{N+j}{\alpha}-1} \theta_{j}(\xi, y) \int_{-i \infty}^{i \infty} d p e^{p x} \frac{1}{K(p)+\xi} \frac{K(p)}{p^{N+j}} \\
& +\frac{1}{2 \pi i} \frac{1}{2 \pi i} \int_{-i \infty}^{i \infty} d \xi e^{\xi t} \int_{-i \infty}^{i \infty} d p e^{p x} \frac{Y^{+}}{K(p)+\xi} I^{+}(p, \xi, y),
\end{aligned}
$$

where

(1.6)

$$
I(z, \xi, y)=\frac{1}{2 \pi i} \int_{-i \infty}^{i \infty} \frac{1}{q-z} \frac{1}{Y^{+}} \frac{K_{1}(q)-K(q)}{K_{1}(q)+\xi}\left(e^{-q y}-\sum_{j=1}^{M} \xi^{\frac{N+j}{\alpha}} \frac{\theta_{j}(\xi, y)}{q^{N+j}}\right) d q .
$$

Here and below $M=\left[\frac{\alpha+1}{2}\right], N=[\alpha]+1-\left[\frac{\alpha+1}{2}\right]$,

$$
Y^{ \pm}=e^{\Gamma^{ \pm}} w^{ \pm}
$$

$\Gamma^{+}(p, \xi)$ and $\Gamma^{-}(p, \xi)$ are left and right limiting values of the sectionally analytic function $\Gamma(z, \xi)$ given by the formula

$$
\Gamma(z, \xi)=\frac{1}{2 \pi i} \int_{-i \infty}^{i \infty} \frac{1}{q-z} \ln \left(\frac{K(q)+\xi}{K_{1}(q)+\xi} \prod_{j=1}^{M} \frac{\left(p-k_{j}\right)}{\left(p+k_{j}\right)} \frac{w^{-}}{w^{+}}\right) d q
$$

and

$$
\begin{gathered}
w^{\mp}(z)=z^{\mu} \prod_{j=1}^{M} \frac{1}{\left(z \pm k_{j}\right)^{\mu}}, \\
\mu=\left\{\frac{\alpha}{2 M}\right\}=\frac{\alpha}{2 M}-\left[\frac{\alpha}{2 M}\right], \\
\theta_{j}(\xi, y)=\left(\mathbb{C}^{-1}\left(\begin{array}{c}
e^{-k_{1}(\xi) y} \\
\ldots \\
e^{-k_{M}(\xi) y}
\end{array}\right)\right)_{j},
\end{gathered}
$$


where a constant matrix

$$
\mathbb{C}=\left\|c_{k j}\right\|_{M}^{M}
$$

here $c_{k j}=\left(-e^{i \pi\left[\frac{\alpha+1}{2}\right]}\right)^{\frac{N+j}{\alpha}} \exp \left\{-i \frac{2 \pi}{\alpha} k(N+j)\right\}$ and

$$
k_{j}(\xi)=\left(-\xi e^{-i \pi\left[\frac{\alpha+1}{2}\right]} \exp (i \pi(2 j-1))\right)^{\frac{1}{\alpha}}, j=1 \ldots, M .
$$

All the integrals are understood in the sense of the principal values.

Now we state the main results.

Theorem 1. Let

$$
u_{0} \in \mathbf{L}^{1}\left(\mathbf{R}^{+}\right), f \in \mathbf{L}^{q}\left(0, T ; \mathbf{L}^{1}\left(\mathbf{R}^{+}\right)\right), h_{j} \in \mathbf{L}^{q}\left(\mathbf{R}^{+}\right) \cap \mathbf{C}^{1}\left(\mathbf{R}^{+}\right), j=1, \ldots, N,
$$

with $q>2$. Then the problem (1.1) is well posed in

$$
\mathbf{C}^{0}\left([0, T], \mathbf{H}_{2}^{N} \cap \mathbf{C}^{N}\right) \cap \mathbf{C}^{0}\left((0, T], \mathbf{H}_{2}^{[\alpha]} \cap \mathbf{C}^{[\alpha]}\right) .
$$

Moreover the solution of the problem (1.1) has the following form:

$$
\begin{aligned}
u(x, t) & =\int_{0}^{+\infty} G(x, y, t) u_{0}(y) d y+\int_{0}^{t} \int_{0}^{+\infty} G(x, y, t-\tau) f(y, \tau) d y d \tau \\
& \left.+\sum_{j=1}^{N} \frac{(-1)^{j-1}}{(j-1) !} \int_{0}^{t} h_{j}(\tau) \lim _{p \rightarrow 0} \partial_{p}^{j-1}\left|\partial_{x}^{\alpha}\right| \widehat{G(x, p, t}-\tau\right) d \tau
\end{aligned}
$$

\section{Preliminaries}

In subsequent consideration, we shall frequently have to use certain theorems of the theory of functions of complex variable, the statements of which we now quote. The proofs may be found in any textbook on the theory. Let $L$ be smooth contour and $\phi(q)$ a function of position on it.

Definition 2. The function $\phi(q)$ is said to satisfy on the curve $L$ the Hölder condition, if for two arbitrary points of this curve,

$$
\left|\phi\left(q_{1}\right)-\phi\left(q_{2}\right)\right| \leq C\left|q_{1}-q_{2}\right|^{\lambda},
$$

where $C$ and $\lambda$ are positive numbers.

Theorem 2. Let $\phi(q)$ be a complex function, which obeys the Hölder condition for all finite $q$ and tends to a definite limit $\phi(\infty)$ as $q \rightarrow \infty$, such that for large $q$ the following inequality holds:

$$
|\phi(q)-\phi(\infty)| \leq C|q|^{-\mu}, \mu>0 .
$$

Then Cauchy type integral

$$
F(z)=\frac{1}{2 \pi i} \int_{-i \infty}^{i \infty} \frac{\phi(q)}{q-z} d q
$$

constitutes a function analytic in the left and right semiplanes. Here and below these functions will be denoted $F^{+}(z)$ and $F^{-}(z)$, respectively. These functions have the limiting values $F^{+}(p)$ and $F^{-}(p)$ at all points of imaginary axis $\operatorname{Re} p=0$, 
on approaching the contour from the left and from the right, respectively. These limiting values are expressed by the Sokhotzki-Plemelj formula,

$$
\begin{aligned}
F^{+}(p) & =\lim _{\substack{z \rightarrow p \\
\operatorname{Re} z<0}} \frac{1}{2 \pi i} \int_{-i \infty}^{i \infty} \frac{\phi(q)}{q-z} \\
& =\frac{1}{2 \pi i} \int_{-i \infty}^{i \infty} \frac{\phi(q)}{q-p} d q+\frac{1}{2} \phi(p), \\
F^{-}(p) & =\lim _{\substack{z \rightarrow p \\
\operatorname{Re} z>0}} \frac{1}{2 \pi i} \int_{-i \infty}^{i \infty} \frac{\phi(q)}{q-z} \\
& =\frac{1}{2 \pi i} \int_{-i \infty}^{i \infty} \frac{\phi(q)}{q-p} d q-\frac{1}{2} \phi(p) .
\end{aligned}
$$

Subtracting and adding the formula (2.1), we obtain the following two equivalent formulas

$$
\begin{aligned}
& F^{+}(p)-F^{-}(p)=\phi(p) \\
& F^{+}(p)+F^{-}(p)=\frac{1}{\pi i} \int_{-i \infty}^{i \infty} \frac{\phi(q)}{q-p} d q
\end{aligned}
$$

which will be employed frequently hereafter. All the integrals are understood in the sense of principal values.

Lemma 1. An arbitrary function $\phi(p)$ given on the contour $\operatorname{Re} p=0$, satisfying the Hölder condition can be uniquely represented in the form

$$
\phi(p)=U^{+}(p)-U^{-}(p),
$$

where $U^{ \pm}(p)$ are the boundary values of the analytic functions $U^{ \pm}(z)$ and the condition $U^{ \pm}(\infty)=0$ holds. These functions are determined by the formula,

$$
U(z)=\frac{1}{2 \pi i} \int_{-i \infty}^{i \infty} \frac{1}{q-z} \phi(q) d q .
$$

Lemma 2. An arbitrary function $\varphi(p)$ given on the contour $\operatorname{Re} p=0$, satisfying the Hölder condition and having zero index,

$$
\operatorname{ind} \varphi(t):=\frac{1}{2 \pi i} \int_{-i \infty}^{i \infty} d \ln \varphi(p)=0,
$$

is uniquely representable as the ratio of the functions $X^{+}(p)$ and $X^{-}(p)$, constituting the boundary values of functions, $X^{+}(z)$ and $X^{-}(z)$, analytic in the left and right complex semiplane and having no zero in these domains. These functions are determined to within an arbitrary constant factor and given by the formula

$$
X^{ \pm}(z)=e^{\Gamma^{ \pm}(z)}, \quad \Gamma(z)=\frac{1}{2 \pi i} \int_{-i \infty}^{i \infty} \frac{1}{q-z} \ln \varphi(q) d q .
$$

Lemma 3. If $L$ is a smooth closed contour and $\phi(q)$ a function that satisfies the Hölder condition on L, then the limiting values of the Cauchy type integral

$$
\Phi(z)=\frac{1}{2 \pi i} \int_{L} \frac{1}{q-z} \phi(q) d q
$$

also satisfy this condition. 


\section{Method of SOlution: Uniqueness theorem}

In this section we get a closed form of the solution of the initial-boundary value problem (1.1) and prove uniqueness of solution.

We denote by $k_{j}(\xi)=K_{1}^{-1}(-\xi)$ the inverse functions for $K_{1}(p)=e^{i \pi\left[\frac{\alpha+1}{2}\right]} q^{\alpha}$. There exist $M=\left[\frac{\alpha+1}{2}\right]$ "positive" inverse functions, such that

$$
\operatorname{Re} k_{j}(\xi)>0
$$

for all $\operatorname{Re} \xi>0$. We will prove below the number of boundary data which we need to put in the problem (1.1) $N=[\alpha]+1-\left[\frac{\alpha+1}{2}\right]$.

Definition 3. We call the function $\mathcal{J}(p, t)$

$$
\mathcal{J}(p, t)=\widehat{u}_{0}(p)+\int_{0}^{t} e^{K_{1}(p) \tau}\left(\widehat{f}(p, \tau)+K_{1}(p) \sum_{l=1}^{N} h_{l}(\tau) p^{-l}\right) d \tau
$$

the input function for problem (1.1).

We denote an $M \times M$ matrix $A(\xi)=\left(a_{l j}(\xi)\right)_{1 \leq l, j \leq M}$ with elements

$$
a_{l j}(\xi)=-\xi k_{l}^{-N-j}(\xi) .
$$

We define the operator $\mathbb{A}$ as

$$
\mathbb{A} \overrightarrow{\mathcal{J}}=\frac{1}{2 \pi i} \int_{-i \infty}^{i \infty} e^{\xi t} A^{-1} \overrightarrow{\mathcal{J}} d \xi
$$

where the input vector

$$
\overrightarrow{\mathcal{J}}=-\left(\mathcal{J}\left(k_{1},+\infty\right), \mathcal{J}\left(k_{2},+\infty\right), \ldots, \mathcal{J}\left(k_{M},+\infty\right)\right)^{T} .
$$

We use a norm

$$
\|\mathbb{A} \overrightarrow{\mathcal{J}}\|=\sum_{j=1}^{m}\left|(\mathbb{A} \overrightarrow{\mathcal{J}})_{j}\right|
$$

Theorem 3. Let

$$
u_{0} \in \mathbf{L}^{1}\left(\mathbf{R}^{+}\right), f \in \mathbf{L}^{q}\left(0, T ; \mathbf{L}^{1}\left(\mathbf{R}^{+}\right)\right), h_{j} \in \mathbf{L}^{q}\left(\mathbf{R}^{+}\right) \cap \mathbf{C}^{1}\left(\mathbf{R}^{+}\right), j=1, \ldots, N,
$$

with $q>2$. Suppose that there exists the solution $u(x, t)$ of the problem (1.1)

$$
u(x, t) \in \mathbf{C}^{0}\left([0, T], \mathbf{H}_{2}^{N} \cap \mathbf{C}^{N}\right) \cap \mathbf{C}^{0}\left((0, T], \mathbf{H}_{2}^{[\alpha]} \cap \mathbf{C}^{[\alpha]}\right) .
$$

Then this solution is unique.

Proof. To derive an integral representation for the solutions of problem (1.1), we suppose that there exists a solution $u(x, t)$ of problem (1.1) which is continued by zero outside of $x>0$ :

$$
u(x, t)=0 \quad \text { for all } \quad x<0 .
$$

Let $\phi(p)$ be a function of the complex variable $p$, which obeys the Hölder condition for all finite $p$ and tends to 0 as $p \rightarrow \pm i \infty$. We define the operator

$$
\mathbb{P} \phi(z)=\mathbb{P}_{p \rightarrow z}\{\phi(p)\}=-\frac{1}{2 \pi i} \int_{-i \infty}^{i \infty} \frac{1}{p-z} \phi(p) d p .
$$

Since the operator $\mathbb{P}$ is defined by a Cauchy type integral, it is readily observed that $\mathbb{P} \phi(z)$ constitutes a function analytic in the entire complex plane, except for 
points of the contour of integration $\operatorname{Re} z=0$. Also by the Sokhotzki-Plemelj formula, we have for $\operatorname{Re} q=0$

$$
\begin{aligned}
& \mathbb{P}_{p \rightarrow q}^{+}\{\phi(p)\}=-\frac{1}{2 \pi i} f_{-i \infty}^{i \infty} \frac{1}{p-q} \phi(p) d p-\frac{1}{2} \phi(q), \\
& \mathbb{P}_{p \rightarrow q}^{-}\{\phi(p)\}=-\frac{1}{2 \pi i} f_{-i \infty}^{i \infty} \frac{1}{p-q} \phi(p) d p+\frac{1}{2} \phi(q) .
\end{aligned}
$$

Here $\mathbb{P}_{p \rightarrow q}^{+}\{\phi(p)\}$ and $\mathbb{P}_{p \rightarrow q}^{-}\{\phi(p)\}$ are limits of $\mathbb{P}_{p \rightarrow z}\{\phi(p)\}$ as $z$ tends to $p$ from the left and right semiplane, respectively.

For the Laplace transform we have

$$
\mathcal{L}_{x \rightarrow q}\left\{\left|\partial_{x}\right|^{\alpha}\right\}=\mathbb{P}_{p \rightarrow q}\left\{|p|^{\alpha}\left(\mathcal{L}_{x \rightarrow p}\{u\}-\sum_{j=1}^{[\alpha]+1} \frac{\partial_{x}^{j-1} u(0, t)}{p^{j}}\right)\right\} .
$$

Since $\mathcal{L}_{x \rightarrow q}\{u\}$ is analytic for all $\operatorname{Re} q>0$, we have

$$
\widehat{u}(q, t)=\mathcal{L}_{x \rightarrow q}\{u\}=\mathbb{P}_{p \rightarrow q}\{\widehat{u}(p, t)\} .
$$

Therefore applying the Laplace transform with respect to $x$ to problem (1.1), we obtain for $t>0$

$$
\left\{\begin{array}{c}
\mathbb{P}_{p \rightarrow q}\left\{\widehat{u}_{t}+K(p) \widehat{u}(p, t)-K(p) \sum_{j=1}^{[\alpha]+1} \frac{\partial_{x}^{j-1} u(0, t)}{p^{j}}-\widehat{f}(p, t)\right\}=0, \\
\widehat{u}(p, 0)=\widehat{u}_{0}(p)
\end{array}\right.
$$

where

We rewrite (3.4) in the form

$$
\left\{\begin{array}{c}
\widehat{u}_{t}+K(p) \widehat{u}(p, t)-K(p) \sum_{j=1}^{[\alpha]+1} \frac{\partial_{x}^{j-1} u(0, t)}{p^{j}}-\widehat{f}(p, t)=\Phi(p, t), \\
\widehat{u}(p, 0)=\widehat{u}_{0}(p),
\end{array}\right.
$$

with some function $\Phi(p, t)$ such that for all Re $p>0$,

$$
\mathbb{P}_{p \rightarrow q}\{\Phi(p, t)\}=0,
$$

and for $|p|>1$,

$$
|\Phi(p, t)| \leq C \frac{1}{|p|^{\alpha-[\alpha]}} .
$$

Applying the Laplace transformation with respect to time variable to problem (3.5), we find for $\operatorname{Re} p>0$

$$
\widehat{\widehat{u}}(p, \xi)=\frac{1}{K(p)+\xi}\left(\widehat{u}_{0}(p)+\widehat{\widehat{f}}(p, \xi)+K(p) \sum_{j=1}^{[\alpha]+1} \frac{\partial_{x}^{j-1} \widehat{u}(0, \xi)}{p^{j}}+\widehat{\Phi}(p, \xi)\right),
$$

where $\widehat{\widehat{u}}(p, \xi)=\mathcal{L}_{t \rightarrow \xi}\{\widehat{u}(p, t)\}$. Here the functions $\widehat{\Phi}(p, \xi), \partial_{x}^{j-1} \widehat{u}(0, \xi), j=1, \ldots,[\alpha]$ +1 are the Laplace transforms for $\Phi(p, t), \partial_{x}^{j-1} \widehat{u}(0, \xi)$ with respect to time, respectively. We will find the function $\widehat{\Phi}(p, \xi)$ using the analytic properties of function $\widehat{\widehat{u}}$ in the right-half complex planes $\operatorname{Re} p>0$ and $\operatorname{Re} \xi>0$. We have for $\operatorname{Re} p=0$

$$
\widehat{\widehat{u}}(p, \xi)=-\frac{1}{\pi i} \int_{-i \infty}^{i \infty} \frac{1}{q-p} \widehat{\widehat{u}}(q, \xi) d q .
$$


In view of the Sokhotzki-Plemelj formula via (3.7), the condition (3.8) can be written as

$$
\Theta^{+}(p, \xi)=-\Lambda^{+}(p, \xi),
$$

where the sectionally analytic functions $\Theta(z, \xi)$ and $\Lambda(z, \xi)$ are given by Cauchy type integrals

$$
\Theta(z, \xi)=\frac{1}{2 \pi i} \int_{-i \infty}^{i \infty} \frac{1}{q-z} \frac{1}{K(q)+\xi} \widehat{\Phi}(q, \xi) d q
$$

and

$$
\Lambda(z, \xi)=\frac{1}{2 \pi i} \int_{-i \infty}^{i \infty} \frac{1}{q-z} \frac{1}{K(q)+\xi}\left(\widehat{u}_{0}(q)+\widehat{\widehat{f}}(p, \xi)+K(q) \sum_{j=1}^{[\alpha]+1} \frac{\partial_{x}^{j-1} \widehat{u}(0, \xi)}{p^{j}}\right) d q
$$

To perform the condition (3.9) in the form of a nonhomogeneous Riemann problem, we introduce the sectionally analytic function

$$
\Omega(z, \xi)=\frac{1}{2 \pi i} \int_{-i \infty}^{i \infty} \frac{1}{q-z} \Psi(q, \xi) d q
$$

where

$$
\Psi(p, \xi)=\frac{K(p)}{K(p)+\xi} \widehat{\Phi}(p, \xi) .
$$

Taking into account the assumed condition (3.6) and making use of SokhotzkiPlemelj formula (2.1), we get for limiting values of the functions $\Omega(z, \xi)$ and $\Theta(z, \xi)$

$$
\Omega^{-}(p, \xi)=-\xi \Theta^{-}(p, \xi) .
$$

Also observe that from (3.10) and (3.12) by formula (2.2),

$$
K(p)\left(\Theta^{+}(p, \xi)-\Theta^{-}(p, \xi)\right)=\Psi(p, \xi)=\Omega^{+}(p, \xi)-\Omega^{-}(p, \xi) .
$$

Substituting (3.9) and (3.14) into this equation, we obtain the nonhomogeneous Riemann problem

$$
\Omega^{+}(p, \xi)=\frac{K(p)+\xi}{\xi} \Omega^{-}(p, \xi)-K(p) \Lambda^{+}(p, \xi) .
$$

It is required that we find two functions for some fixed point $\xi, \operatorname{Re} \xi>0: \Omega^{+}(z, \xi)$ analytic in $\operatorname{Re} z<0$ and $\Omega^{-}(z, \xi)$ analytic in $\operatorname{Re} z>0$, which satisfy on the contour Re $p=0$ the relation (3.15).

Here, for some fixed point $\xi$, Re $\xi>0$, the functions

$$
W(p, \xi)=\frac{K(p)+\xi}{\xi} \text { and } g(p, \xi)=-K(p) \Lambda^{+}(p, \xi)
$$

are called the coefficient and the free term of the Riemann problem, respectively.

Bearing in mind formula (3.13), note that we can find an unknown function $\widehat{\Phi}(p, \xi)$ which is involved in the formula (3.7) by the relation

$$
\widehat{\Phi}(p, \xi)=\frac{K(p)+\xi}{K(p)}\left(\Omega^{+}(p, \xi)-\Omega^{-}(p, \xi)\right) .
$$

The method for solving the Riemann problem $A^{+}(p)=\varphi(p) A^{-}(p)+\phi(p)$ is based on Lemmas 1 and 2 
In the formulations of Lemmas 1 and 2, the coefficient $\varphi(p)$ and the free term $\phi(p)$ of the Riemann problem are required to satisfy the Hölder condition on the contour $\operatorname{Re} p=0$. This restriction is essential. On the other hand, it is easy to observe that both functions $W(p, \xi)$ and $g(p, \xi)$ do not have a limiting value as $p \rightarrow \pm i \infty$. So we cannot find the solution using $\ln W(p, \xi)$. The principal task now is to get an expression equivalent to the boundary value problem (3.15), such that the conditions of Lemmas 1 and 2 are satisfied. First, let us introduce some notation and let us establish certain auxiliary relationships. Denote

$$
K_{1}(z)=C_{\alpha} z^{\alpha}, C_{\alpha}=e^{i \pi\left[\frac{\alpha+1}{2}\right]}
$$

We choose the constant $e^{i \pi\left[\frac{\alpha+1}{2}\right]}$ such that $\operatorname{Re} K_{1}(q)>0$ for $\operatorname{Re} q=0$. The equation $K_{1}(p)=-\xi$ has $M=\left[\frac{\alpha+1}{2}\right]$ roots $k_{1}(\xi), k_{2}(\xi), \ldots, k_{M}(\xi)$, which are analytic functions for $\operatorname{Re} \xi>0$ and

$$
p=k_{j}(\xi)=\left(\frac{\xi}{C_{\alpha}} \exp (2 i \pi j)\right)^{\frac{1}{\alpha}}
$$

transforms the half-complex plane $\operatorname{Re} \xi>0$ to domains, where

$$
\operatorname{Re} k_{j}(\xi)>0, j=1,2, \ldots, M .
$$

We introduce the function

$$
\widetilde{W}(p, \xi)=\left(\frac{K(p)+\xi}{K_{1}(p)+\xi}\right) \frac{w^{-}}{w^{+}} \prod_{j=1}^{M} \frac{\left(p-k_{j}\right)}{\left(p+k_{j}\right)} \neq 0
$$

where

$$
\begin{gathered}
w^{-}(z)=z^{\mu}\left(\prod_{j=1}^{M} \frac{1}{z+k_{j}(\xi)}\right)^{\mu}, w^{+}=z^{\mu}\left(\prod_{j=1}^{M} \frac{1}{z-k_{j}(\xi)}\right)^{\mu}, \\
\mu=\left\{\frac{\alpha}{2 M}\right\} .
\end{gathered}
$$

Here $w^{-}(z)$ is analytic for $\operatorname{Re} z>0$ and $w^{+}(z)$ is analytic for $\operatorname{Re} z<0$.

We observe that the function $\widetilde{W}(p, \xi)$, given on the contour Re $p=0$, satisfies the Hölder condition, and under the assumption $\operatorname{Re} K_{1}(p)>0$ does not vanish for any $\operatorname{Re} \xi>0$. Also we have

$$
\text { Ind. } \widetilde{W}(p, \xi)=\frac{1}{2 \pi i} \int_{-i \infty}^{i \infty} d \ln \widetilde{W}(p, \xi)=0 .
$$

Therefore in accordance with Lemma 2 the function $\widetilde{W}(p, \xi)$ can be represented in the form of the ratio

$$
\widetilde{W}(p, \xi)=\frac{X^{+}(p, \xi)}{X^{-}(p, \xi)}
$$

where

$$
X^{ \pm}(p, \xi)=e^{\Gamma^{ \pm}(p, \xi)}, \quad \Gamma(z, \xi)=\frac{1}{2 \pi i} \int_{-i \infty}^{i \infty} \frac{1}{q-z} \ln \widetilde{W}(q, \xi) d q
$$


Now we return to the nonhomogeneous Riemann problem (3.15). Making use of the formula (3.17), we get

$$
\frac{K(p)+\xi}{K_{1}(p)+\xi}=\frac{Y^{+}(p, \xi)}{Y^{-}(p, \xi)} \prod_{j=1}^{M} \frac{\left(p+k_{j}\right)}{\left(p-k_{j}\right)}
$$

where

$$
Y^{ \pm}(p, \xi)=X^{ \pm}(p, \xi) w^{ \pm}(p) .
$$

By (3.18) we reduce the nonhomogeneous Riemann problem (3.15) to the form

$$
\frac{1}{Y^{+}} \Omega^{+}(p, \xi)=\left(\frac{K_{1}(p)+\xi}{\xi}\right) \prod_{j=1}^{M} \frac{\left(p+k_{j}\right)}{\left(p-k_{j}\right)} \frac{\Omega^{-}(p, \xi)}{Y^{-}(p, \xi)}-\frac{1}{Y^{+}(p, \xi)} K(p) \Lambda^{+}(p, \xi) .
$$

Now we perform the function $\Lambda(z, \xi)$ given by formula (3.11) as

$$
\Lambda(z, \xi)=\Lambda_{1}(z, \xi)+\Lambda_{2}(z, \xi),
$$

where

$$
\begin{aligned}
\Lambda_{1}(z, \xi)= & \frac{1}{2 \pi i} \int_{-i \infty}^{i \infty} \frac{1}{q-z} \frac{1}{K_{1}(q)+\xi} \\
& \times\left(\widehat{u}_{0}(q)+\widehat{\widehat{f}}(q, \xi)+K_{1}(q) \sum_{j=1}^{[\alpha]+1} \frac{\partial_{x}^{j-1} \widehat{u}(0, \xi)}{p^{j}}\right) d q, \\
\Lambda_{2}(z, \xi)= & \frac{1}{2 \pi i} \int_{-i \infty}^{i \infty} \frac{1}{q-z} \frac{K_{1}(q)-K(q)}{(K(q)+\xi)\left(K_{1}(q)+\xi\right)} \\
& \times\left(\widehat{u}_{0}(q)+\widehat{\widehat{f}}(q, \xi)-\xi \sum_{j=1}^{[\alpha]+1} \frac{\partial_{x}^{j-1} \widehat{u}(0, \xi)}{p^{j}}\right) d q .
\end{aligned}
$$

First we calculate the left limiting value $\Lambda_{1}^{+}(p, \xi)$. There exist $M$ roots $k_{j}(\xi)$ of equation $K_{1}(z)=-\xi$ such that $\operatorname{Re} k_{j}(\xi)>0$ for all $\operatorname{Re} \xi>0$. Therefore, taking the limit $z \rightarrow p$ from the left-hand side of the complex plane, by the Cauchy theorem we get

$$
\Lambda_{1}^{+}(p, \xi)=\sum_{j=1}^{M}-\frac{k_{j}^{\prime}(\xi)}{p-k_{j}(\xi)}\left(\widehat{u}_{0}\left(k_{j}\right)+\widehat{\widehat{f}}\left(k_{j}, \xi\right)-\xi \sum_{l=1}^{[\alpha]+1} \frac{\partial_{x}^{l-1} \widehat{u}(0, \xi)}{k_{j}^{l}}\right) .
$$

The last relation implies that $\left(K_{1}(p)+\xi\right) \Lambda_{1}^{+}(p, \xi)$ can be expressed by the function $\Lambda_{3}(z, \xi)$ which is analytic in $\operatorname{Re} z>0$,

$$
\left(K_{1}(p)+\xi\right) \Lambda_{1}^{+}(p, \xi)=\Lambda_{3}^{-}(p, \xi),
$$

where

$$
\Lambda_{3}(z, \xi)=\left(K_{1}(p)+\xi\right) \sum_{j=1}^{M}-\frac{k_{j}^{\prime}(\xi)}{p-k_{j}(\xi)}\left(\widehat{u}_{0}\left(k_{j}\right)+\widehat{\widehat{f}}\left(k_{j}, \xi\right)-\xi \sum_{l=1}^{[\alpha]+1} \frac{\partial_{x}^{l-1} \widehat{u}(0, \xi)}{k_{j}^{l}}\right) .
$$

By the Sokhotzki-Plemelj formula (2.2) we express the left limiting value $\Lambda_{2}^{+}(p, \xi)$ in the term of the right limiting value $\Lambda_{2}^{-}(p, \xi)$ as

$$
\Lambda_{2}^{+}(p, \xi)=\Lambda_{2}^{-}(p, \xi)+\widetilde{g}_{1}(p, \xi),
$$


where

$$
\widetilde{g}_{1}(p, \xi)=\frac{K_{1}(p)-K(p)}{(K(p)+\xi)\left(K_{1}(p)+\xi\right)}\left(\widehat{u}_{0}(q)+\widehat{\widehat{f}}(q, \xi)-\xi \sum_{j=1}^{[\alpha]+1} \frac{\partial_{x}^{j-1} \widehat{u}(0, \xi)}{p^{j}}\right) .
$$

Bearing in mind the representation (3.20) and making use of (3.23), (3.25) and (3.18), after simple transformations we get

$$
K(p) \Lambda^{+}=(K(p)+\xi)\left[\frac{\Lambda_{3}^{-}}{K_{1}(p)+\xi}+\Lambda_{2}^{-}\right]-\xi \Lambda^{+}+g_{1}(p, \xi)
$$

where

$$
g_{1}(p, \xi)=(K(p)+\xi) \widetilde{g}_{1}(p, \xi) .
$$

Replacing in equation (3.19) $K(p) \Lambda^{+}(p, \xi)$ by (3.26), we reduce the nonhomogeneous Riemann problem (3.19) in the form

$$
\frac{\Omega^{+}(p, \xi)-\xi \Lambda^{+}(p, \xi)}{Y^{+}(p, \xi)}=\left(\frac{K_{1}(p)+\xi}{\xi}\right) \frac{P_{+}(p, \xi)}{P_{-}(p, \xi)} \frac{\Omega_{1}^{-}(p, \xi)}{Y^{-}(p, \xi)}-\frac{1}{Y^{+}(p, \xi)} g_{1}(p, \xi),
$$

where

$$
\Omega_{1}^{-}(p, \xi)=\Omega^{-}(p, \xi)-\xi \Lambda_{2}^{-}(p, \xi)-\frac{\xi \Lambda_{3}^{-}}{K_{1}(p)+\xi} .
$$

Since $g_{1}(p, \xi)$ satisfies on Re $p=0$ the Hölder condition, on the basis of Lemma 3 the function $\frac{1}{Y^{+}(p, \xi)} g_{1}(p, \xi)$ also satisfies this condition. Therefore in accordance with Lemma 1 it can be uniquely represented in the form of the difference of the functions $U^{+}(p, \xi)$ and $U^{-}(p, \xi)$, constituting the boundary values of the analytic function $U(z, \xi)$, given by formula

$$
\begin{aligned}
U(z, \xi)= & \frac{1}{2 \pi i} \int_{-i \infty}^{i \infty} \frac{1}{q-z} \frac{1}{Y^{+}(q, \xi)} \frac{K_{1}(p)-K(p)}{K_{1}(p)+\xi} \\
& \times\left(\widehat{u}_{0}(q)+\widehat{\widehat{f}}(q, \xi)-\xi \sum_{j=1}^{[\alpha]+1} \frac{\partial_{x}^{j-1} \widehat{u}(0, \xi)}{p^{j}}\right) d q .
\end{aligned}
$$

Therefore the problem (3.19) takes the form

$$
\frac{\Omega^{+}(p, \xi)-\xi \Lambda^{+}(p, \xi)}{Y^{+}(p, \xi)}+U^{+}(p, \xi)=\frac{\Omega_{1}^{-}(p, \xi)}{Y^{-}(p, \xi)}+U^{-}(p, \xi) .
$$

The last relation indicates that the function $\frac{\Omega^{+}(p, \xi)-\xi \Lambda^{+}(p, \xi)}{Y^{+}}+U^{+}$, analytic in $\operatorname{Re} z<0$, and the function $\frac{\Omega_{1}^{-}}{Y^{-}}+U^{-}$, analytic in $\operatorname{Re} z>0$, constitute the analytic continuation of each other through the contour Re $z=0$. Consequently, they are branches of a unique analytic function in the entire plane. According to the Liouville theorem, this function is zero. Thus, bearing in mind the representations (3.28), we get

$$
\begin{aligned}
& \Omega^{+}(p, \xi)=-Y^{+} U^{+}+\xi \Lambda^{+}, \\
& \Omega^{-}(p, \xi)=-\frac{\xi}{K_{1}(p)+\xi} Y^{-} \prod_{j=1}^{M} \frac{\left(p-k_{j}\right)}{\left(p+k_{j}\right)} U^{-}+\xi\left(\Lambda_{1}^{+}+\Lambda_{2}^{-}\right) .
\end{aligned}
$$

There exist $M$ roots $k_{j}(\xi)$ of equation $K_{1}(z)=-\xi$ such that $\operatorname{Re} k_{j}(\xi)>0$ for all $\operatorname{Re} \xi>0$. Therefore, in the expression for the function $\Omega^{-}(z, \xi)$, the function $\xi \Lambda_{1}^{+}$ 
has $M$ poles in the points $z=k_{j}(\xi), j=1, \ldots, M$. Thus in the general case the problem (3.15) is unsolvable. For solubility of the nonhomogeneous problem (3.15), it is necessary and sufficient that the following conditions are satisfied:

$$
\widehat{u}_{0}\left(k_{l}\right)+\widehat{\widehat{f}}\left(k_{l}, \xi\right)-\xi \sum_{j=1}^{[\alpha]+1} \phi_{l}^{-j} \int_{0}^{+\infty} e^{-\xi \tau} u_{x}^{(j-1)}(0, \tau) d \tau=0,
$$

for $l=1,2, \ldots, M$, where

$$
\begin{aligned}
\widehat{u}_{0}\left(k_{l}\right) & =\int_{0}^{+\infty} e^{-\phi_{l} y} u_{0}(y) d y \\
\widehat{\widehat{f}}\left(k_{l}, \xi\right) & =\int_{0}^{+\infty} \int_{0}^{+\infty} e^{-\left(\phi_{l} y+\xi t\right)} f(y, t) d y d t .
\end{aligned}
$$

We have $M$ equations with $[\alpha]+1$ unknowns $u_{x}^{(j-1)}(0, t)$, so we must include $N=[\alpha]+1-M$ boundary data into the problem. For example, if we use Dirichlet type boundary data $u_{x}^{(j-1)}(0, t)=h_{j}(t), j=1,2, \ldots, N$, then we can find the rest of the Laplace transforms of the boundary values

$$
\widehat{v}_{j}(\xi) \equiv \int_{0}^{+\infty} e^{-\xi t} \partial_{x}^{(j+N-1)} u(0, t) d t, j=1, \ldots, M,
$$

from the system of $M$ equations

$$
A \overrightarrow{\hat{V}}=\overrightarrow{\mathcal{J}}
$$

where $A$ is $M \times M$ matrix $A=\left\|a_{i j}\right\|_{M}^{M}$ with elements

$$
a_{i j}=-\xi k_{i}^{-N-j},
$$

the vector $\overrightarrow{\hat{V}}=\left(\widehat{v}_{1}(\xi), \ldots, \widehat{v}_{M}(\xi)\right)^{T}$ and $\overrightarrow{\mathcal{J}}$ is the input characteristic vector for problem (1.1) with components

$$
\mathcal{J}\left(k_{l},+\infty\right)=-\left(\widehat{u}_{0}\left(k_{l}\right)+\widehat{\widehat{f}}\left(k_{l}, \xi\right)\right)+\xi \sum_{j=1}^{N} \widehat{h}_{j}(\xi) k_{l}^{-j} .
$$

Here

$$
\widehat{h}_{j}(\xi)=\int_{0}^{+\infty} e^{-\xi t} h_{j}(t) d t
$$

The determinant of system (3.31) is not equal to zero since all functions $k_{l}, l=$ $1, \ldots, M$ are different for $\operatorname{Re} \xi>0$. Solving (3.31) and taking the inverse Laplace inverse transformation with respect to time, we obtain

$$
\vec{V}(t)=\left(\begin{array}{c}
v_{1}(t) \\
\ldots \\
v_{M}(t)
\end{array}\right)=\left(\begin{array}{c}
\partial_{x}^{(N)} u(0, t) \\
\ldots \\
\partial_{x}^{([\alpha])} u(0, t)
\end{array}\right)=\frac{1}{2 \pi i} \int_{-i \infty}^{i \infty} e^{\xi t} A^{-1} \overrightarrow{\mathcal{J}} d \xi .
$$


Now we return to problem (3.15). Under condition (3.31), $\Lambda_{1}^{+}=0$. Therefore from (3.30) the limiting values of solution of (3.15) are given by formula

$$
\begin{aligned}
& \Omega^{+}(p, \xi)=-Y^{+} U^{+}+\xi \Lambda_{2}^{+} \\
& \Omega^{-}(p, \xi)=-\frac{\xi}{K_{1}(p)+\xi} \prod_{j=1}^{M} \frac{\left(p-k_{j}\right)}{\left(p+k_{j}\right)} Y^{-} U^{-}+\xi \Lambda_{2}^{-} .
\end{aligned}
$$

From (3.34), with the help of the integral representations (3.29) and (3.22), for sectionally analytic functions $U(z, \xi)$ and $\Lambda_{2}(z, \xi)$, making use of the SokhotzkiPlemelj formula (2.1) and relation (3.18), we can express the difference limiting values of the function $\Omega(z, \xi)$ in the form

$$
\begin{aligned}
\Omega^{+}(p, \xi)-\Omega^{-}(p, \xi) & =-Y^{+}\left(U^{+}-\frac{\xi}{K(p)+\xi} U^{-}\right)+\xi\left(\Lambda_{2}^{+}-\Lambda_{2}^{-}\right) \\
& =-Y^{+} \frac{K(p)}{K(p)+\xi} U^{-}-K(p) \frac{1}{K(p)+\xi} g_{1} .
\end{aligned}
$$

We now proceed to find the unknown function $\widehat{\Phi}(p, \xi)$ involved in formula (3.7) for the solution $\widehat{\widehat{u}}(p, \xi)$ of problem (1.1). Replacing the difference $\Omega^{+}(p, \xi)-\Omega^{-}(p, \xi)$ in the relation (3.16) by formula (3.35) we get

$$
\begin{aligned}
\widehat{\Phi}(p, \xi) & =\frac{K(p)+\xi}{K(p)}\left(\Omega^{+}(p, \xi)-\Omega^{-}(p, \xi)\right) \\
& =-Y^{+}(p, \xi) U^{+}
\end{aligned}
$$

It is easy to observe that $\widehat{\Phi}(p, \xi)$ is the boundary value of the function analytic in the left complex semiplane and therefore satisfies our basic assumption for all $\operatorname{Re} z>0$

$$
\mathbb{P}\{\Phi\}=0
$$

Having determined the function $\widehat{\Phi}(p, \xi)$, and bearing in mind formula (3.7) and conditions $\partial_{x}^{j-1} u(0, t)=0, j=1,2, \ldots, N$, we determine required function $\widehat{\widehat{u}}$,

$$
\widehat{\widehat{u}}(p, \xi)=\frac{1}{K(p)+\xi}\left(\widehat{u}_{0}(p)+\widehat{\widehat{f}}(p, \xi)+K(p) \sum_{j=1}^{M} \frac{\partial_{x}^{N+j-1} \widehat{u}(0, \xi)}{p^{N+j}}-Y^{+}(p, \xi) U^{+}\right),
$$

where $\partial_{x}^{N+j-1} \widehat{u}(0, \xi), j=1, \ldots, M$ was defined by formula (3.33). Now we prove that, in accordance with the last relation, the function $\widehat{\widehat{u}}(p, \xi)$ constitutes the limiting value of an analytic function in $\operatorname{Re} z>0$.

With the help of the integral representations (3.29) for the sectionally analytic function $U(z, \xi)$ making use of the Sokhotzki-Plemelj formula (2.1), we arrive at 
the relation

$$
\begin{aligned}
\widehat{\widehat{u}} & =\frac{1}{K_{1}(p)+\xi}\left(\widehat{u}_{0}(p)+\widehat{\widehat{f}}(p, \xi)\right) \\
& +\frac{K_{1}(p)}{K_{1}(p)+\xi}\left(\sum_{j=1}^{N} \frac{\widehat{h}_{j}(\xi)}{p^{N+j}}+\sum_{j=1}^{M} \frac{\partial_{x}^{N+j-1} \widehat{u}(0, \xi)}{p^{N+j}}\right) \\
& -\frac{1}{K_{1}(p)+\xi} \prod_{j=1}^{M} \frac{\left(p-k_{j}\right)}{\left(p+k_{j}\right)} Y^{-} U^{-},
\end{aligned}
$$

where $\partial_{x}^{N+j-1} \widehat{u}(0, \xi), j=1, \ldots, M$ was defined by formula (3.33).

Thus by virtue of (3.31) the function $\widehat{\widehat{u}}$ is the limiting value of an analytic function in $\operatorname{Re} z>0$. Note the fundamental importance of the proven fact - that the solution $\widehat{\widehat{u}}$ constitutes an analytic function in $\operatorname{Re} z>0$ and, as a consequence, its inverse Laplace transform vanishes for all $x<0$.

Taking the inverse Laplace transform of (3.37) with respect to $\xi$ and $p$ variables, we get the closed form for solution $u(x, t)$

$$
\begin{aligned}
u(x, t) & =\frac{1}{2 \pi i} \int_{-i \infty}^{i \infty} e^{p x} e^{-K(p) t} \mathcal{J}(p, t) d p \\
& +\frac{1}{2 \pi i} \int_{-i \infty}^{i \infty} d p e^{p x} K_{1}(p) \sum_{j=1}^{M} p^{-N-j} \int_{0}^{t} e^{-K(p)(t-\tau)} v_{j}(\tau) d \tau \\
& -\frac{1}{2 \pi i} \int_{-i \infty}^{i \infty} e^{p x} \widehat{u}_{1}(p, t),
\end{aligned}
$$

where

$$
\begin{aligned}
& v_{j}(t)=\partial_{x}^{N+j-1} \widehat{u}(0, \xi)=\frac{1}{2 \pi i} \int_{-i \infty+b}^{i \infty+b} e^{\xi t}\left(A^{-1} \overrightarrow{\mathcal{J}}\right)_{j}(\xi) d \xi=(\mathbb{A} \overrightarrow{\mathcal{J}})_{j} \\
& \widehat{u}_{1}(p, t)=-\frac{1}{2 \pi i} \int_{-i \infty}^{i \infty} e^{\xi t} \frac{1}{K_{1}(p)+\xi} \prod_{j=1}^{M} \frac{\left(p-k_{j}\right)}{\left(p+k_{j}\right)} Y^{-}(p, \xi) U^{-}(p, \xi) d \xi
\end{aligned}
$$

We now prove the uniqueness of the solution. On the contrary, we consider two different solutions $u_{1}$ and $u_{2}$. The difference $u_{1}-u_{2}$ then satisfies the linear problem (1.1) with homogeneous data $f=0, u_{0}=0$, and $h_{j}=0$. Then by (3.38) we get $u_{1}-u_{2}=0$; hence $u_{1}=u_{2}$. The theorem is proved.

\section{Green operator}

We introduce the operator $\mathbb{G}$

$$
\mathbb{G}(t) \phi(x)=\int_{0}^{+\infty} \phi(y) G(x, y, t) d y
$$

and the operator $\mathcal{H}(t)$

$$
\mathcal{H}(t) \vec{h}=\sum_{j=1}^{N} \frac{(-1)^{j-1}}{(j-1) !} \int_{0}^{t} h_{j}(\tau) \lim _{p \rightarrow 0} \partial_{p}^{j-1}\left|\partial_{x}^{\alpha}\right| G(\widehat{x, p, t}-\tau) d \tau
$$


where the function $G(x, y, t)$ is defined by (1.5). Now we collect some preliminary estimates of the operators $\mathbb{G}(t) \phi(x)$ and $\mathcal{H}(t) \vec{h}$.

Lemma 4. The estimates are true, provided that the right-hand sides are finite

$$
\begin{aligned}
\left\|\partial_{x}^{n} \mathbb{G}(t) \phi\right\|_{\mathbf{L}^{s}} & \leq C t^{-\frac{1}{\alpha}\left(\frac{1}{r}-\frac{1}{s}+n\right)}\|\phi(\cdot)\|_{\mathbf{L}^{r}} \\
\left\|\partial_{x}^{n} \mathcal{H}(t) \vec{h}\right\|_{\mathbf{L}^{s}} & \leq C\left(\left\|\vec{h}^{\prime}(\cdot)\right\|_{L^{\infty}}+\|\vec{h}(\cdot)\|_{L^{\infty}}\right) t^{-\frac{N-n}{\alpha}},
\end{aligned}
$$

where small $\mu>0,1 \leq r \leq s \leq \infty, n=0,1, \ldots,[\alpha]$.

Proof. From definition (1.5) we have

$$
G(x, y, t)=\sum_{j=1}^{4} J_{j}(x, y, t),
$$

where

$$
\begin{gathered}
J_{1}(x, y, t)=\frac{1}{2 \pi i} \int_{-i \infty}^{i \infty} e^{p x-K(p) t} e^{-p y} d p \\
J_{2}(x, y, t)=-\frac{1}{2 \pi i} \frac{1}{2 \pi i} \int_{-i \infty}^{i \infty} d \xi e^{\xi t} \int_{-i \infty}^{i \infty} e^{p x} \frac{K(p)}{\xi(K(p)+\xi)} \sum_{j=1}^{M} \frac{\theta_{j}(\xi, y) \xi^{\frac{N+j}{\alpha}}}{p^{N+j}} d p \\
J_{3}(x, y, t)=-\frac{1}{2 \pi i} \frac{1}{2 \pi i} \int_{-i \infty}^{i \infty} d \xi e^{\xi t} \int_{-i \infty}^{i \infty} e^{p x} \frac{Y^{+}(p, \xi)}{K(p)+\xi} I_{1}^{+}(p, \xi, y) d p \\
I_{1}(z, \xi, y)=\frac{1}{2 \pi i} \int_{-i \infty}^{i \infty} \frac{e^{-q y}}{q-z} \frac{1}{Y^{+}} \frac{K_{1}(q)-K(q)}{K_{1}(q)+\xi} d q
\end{gathered}
$$

and

$$
\begin{aligned}
& J_{4}(x, y, t)=-\frac{1}{2 \pi i} \frac{1}{2 \pi i} \int_{-i \infty}^{i \infty} d \xi e^{\xi t} \int_{-i \infty}^{i \infty} e^{p x} \frac{Y^{+}(p, \xi)}{K(p)+\xi} I_{2}^{+}(p, \xi, y) d p \\
& I_{2}(z, \xi, y)=\frac{1}{2 \pi i} \int_{-i \infty}^{i \infty} \frac{1}{q-z} \frac{1}{Y^{+}} \frac{K_{1}(q)-K(q)}{K_{1}(q)+\xi} \sum_{j=1}^{M} \xi^{\frac{N+j}{\alpha}} \frac{\theta_{j}(\xi, y)}{q^{N+j}} d q .
\end{aligned}
$$

Denote by

$$
\mathcal{J}_{j}(t) \phi=\theta(x) \int_{0}^{+\infty} J_{j}(x, y, t) \phi(y) d y,
$$

a similar consideration to that in the book $[\underline{3}$, which proves that for $n=0,1, \ldots,[\alpha]$,

$$
\left\|\partial_{x}^{n} \mathcal{J}_{1}(t) \phi\right\|_{\mathbf{L}^{s}} \leq C t^{-\frac{1}{\alpha}\left(\frac{1}{r}-\frac{1}{s}\right)-\frac{n}{\alpha}}\|\phi(\cdot)\|_{\mathbf{L}^{r}} .
$$

Also in the book [4] it was proved that

$$
\left\|\partial_{x}^{n} \mathcal{J}_{2}(t) \phi\right\|_{\mathbf{L}^{s, \mu}} \leq C t^{-\frac{1}{\alpha}\left(\frac{1}{r}-\frac{1}{s}\right)-\frac{n}{\alpha}}\|\phi(\cdot)\|_{\mathbf{L}^{r}} .
$$

Now we estimate $\mathcal{J}_{2}(t) \phi$. Let the contours $\mathcal{C}_{i}$ be defined as

$$
\begin{aligned}
& \mathcal{C}_{1}=\left\{p \in\left(\infty e^{-i\left(\frac{\pi}{2}+\varepsilon_{1}\right)}, 0\right) \bigcup\left(0, \infty e^{i\left(\frac{\pi}{2}+\varepsilon_{1}\right)}\right)\right\}, \\
& \mathcal{C}_{2}=\left\{q \in\left(\infty e^{-i\left(\frac{\pi}{2}-\varepsilon_{2}\right)}, 0\right) \bigcup\left(0, \infty e^{i\left(\frac{\pi}{2}-\varepsilon_{2}\right)}\right)\right\}, \\
& \mathcal{C}_{3}=\left\{\xi \in\left(\infty e^{-i\left(\frac{\pi}{2}+\varepsilon_{3}\right)}, 0\right) \bigcup\left(0, \infty e^{i\left(\frac{\pi}{2}+\varepsilon_{3}\right)}\right)\right\},
\end{aligned}
$$


where $\varepsilon_{j}>0$ small enough can be chosen such that all functions under integration are analytic and $\operatorname{Re} k_{j}(\xi)>0$ for $\xi \in \mathcal{C}_{3}$. In particular, for example, $K_{1}(p)+\xi \neq 0$ outside the origin for all $p \in \mathcal{C}_{1}$ and $\xi \in \mathcal{C}_{3}$.

From the integral representation (4.4) we have for $y>0, \xi \in \mathcal{C}_{3}$, and $p \in \mathcal{C}_{1}$,

$$
\begin{aligned}
& I_{1}^{+}(z, \xi, y)=\frac{1}{2 \pi i} \int_{-i \infty}^{i \infty} \frac{e^{-q y}}{q-z} \frac{1}{Y^{+}} \frac{K_{1}(q)-K(q)}{K_{1}(q)+\xi} d q \\
& =\frac{1}{2 \pi i} \int_{\mathcal{C}_{2}} \frac{e^{-q y}}{q-p}\left(\frac{1}{Y^{-}(q, \xi)} \frac{P_{+}(q, \xi)}{P_{-}(q, \xi)} \frac{K_{1}(q)-K(q)}{K(q)+\xi}-1\right) d q .
\end{aligned}
$$

Here

$$
K(p)=\left\{\begin{aligned}
(-i p)^{\alpha}, & \operatorname{Im} p>0 \\
(i p)^{\alpha}, & \operatorname{Im} p<0 .
\end{aligned}\right.
$$

Since $\Gamma(z, \xi)=O\left(\frac{\xi}{\langle z\rangle}\right)$, we have

$$
\frac{1}{Y^{-}(q, \xi)} \frac{P_{+}(q, \xi)}{P_{-}(q, \xi)} \frac{K_{1}(q)-K(q)}{K(q)+\xi}-1=O\left(\xi\langle q\rangle^{-1}\right) .
$$

Substituting the obtained relation into (4.10), we get

$$
\left|I_{1}^{+}(z, \xi, y)\right| \leq C|\xi| \int_{\mathcal{C}_{2}} \frac{e^{-C|q| y}}{|q-p|}\{q\}^{-\mu+\alpha}\langle q\rangle^{-1} d q .
$$

After this observation in accordance with the integral representation (4.3) by the Holder inequality we have arrived at the following estimate for $r>1, s>1, l^{-1}=$ $1-r^{-1}$, small $\mu \geq 0, n=0,1, \ldots,[\alpha]$ :

$$
\begin{aligned}
& \left\|\partial_{x}^{n} \int_{0}^{+\infty} J_{3}(\cdot, y, t) \phi(y) d y\right\|_{\mathbf{L}^{s}} \\
& \leq C \int_{\mathcal{C}_{3}} d \xi e^{-C|\xi| t} \int_{\mathcal{C}_{1}} d p \frac{|p|^{n-\frac{1}{s}}}{|K(p)+\xi|} \\
& \times \int_{\mathcal{C}_{2}} \frac{1}{|q-p|} d q\left\langle|\xi|^{-\frac{1}{\alpha}} q\right\rangle^{-1}\left\|e^{-C|q| \cdot}\right\|_{\mathbf{L}^{l}}\|\phi\|_{\mathbf{L}^{r}} \\
& \leq C\|\phi\|_{\mathbf{L}^{r}} \int_{\mathcal{C}_{3}} d \xi e^{-C|\xi| t} \int_{\mathcal{C}_{1}} d p \frac{|p|^{n-\frac{1}{s}}}{|K(p)+\xi|} \int_{\mathcal{C}_{2}} d q|q|^{\frac{1}{r}-1}\left\langle|\xi|^{-\frac{1}{\alpha}} q\right\rangle^{-1} \frac{1}{|q-p|} \\
& \leq C\|\phi\|_{\mathbf{L}^{r}} t^{-\frac{1}{\alpha}\left(\frac{1}{r}-\frac{1}{s}+n\right)} .
\end{aligned}
$$

Since $\left|\theta_{j}(\xi, y)\right| \leq C e^{-C|\xi|^{\frac{1}{\alpha}} y}$, we have

$$
\left.I_{2}^{+}(z, \xi, y) \leq C|\xi| e^{-C|\xi|^{\frac{1}{\alpha}} y} \int_{\mathcal{C}_{2}} \frac{1}{|q-p|}\{q\}^{-\mu+\{\alpha\}}\langle q\rangle^{-N-1} d q\right) .
$$


Therefore

$$
\begin{aligned}
& \left\|\partial_{x}^{n} \int_{0}^{+\infty} J_{4}(\cdot, y, t) \phi(y) d y\right\|_{\mathbf{L}^{s}} \\
& \leq C \int_{\mathcal{C}_{3}} d \xi e^{-C|\xi| t}|\xi| \int_{\mathcal{C}_{1}} d p \frac{|p|^{n-\frac{1}{s}}}{|K(p)+\xi|} \\
& \times \int_{\mathcal{C}_{2}} \frac{1}{|q-p|} d q\{q\}^{-\mu+\{\alpha\}}\langle q\rangle^{-N-1}\left\|e^{-C|\xi|^{\frac{1}{\alpha}}}\right\|_{\mathbf{L}^{l}}\|\phi\|_{\mathbf{L}^{r}} \\
& \leq C\|\phi\|_{\mathbf{L}^{r}} \int_{\mathcal{C}_{3}} d \xi e^{-C|\xi| t}|\xi|^{\frac{1}{\alpha}\left(\frac{1}{r}-1\right)} \\
& \times \int_{\mathcal{C}_{1}} d p \frac{|p|^{n-\frac{1}{s}}}{|K(p)+\xi|} \int_{\mathcal{C}_{2}} d q\{q\}^{-\mu+\{\alpha\}}\langle q\rangle^{-N-1} \frac{1}{|q-p|} \\
& \leq C\|\phi\|_{\mathbf{L}^{r}} t^{-\frac{1}{\alpha}\left(\frac{1}{r}-\frac{1}{s}+n\right)} .
\end{aligned}
$$

Therefore according to (4.5)-(4.12) we obtain the first part of the estimate (4.1) of Lemma 4

Now we prove the second part of the estimate (4.1) of Lemma 4. Denote

$$
\tilde{\theta}_{j}(\xi, y)=\left(\mathbb{C}^{-1}\left(\begin{array}{c}
k_{1}^{-j}(\xi) \\
\ldots \\
k_{M}^{-j}(\xi)
\end{array}\right)\right)_{j},
$$

where a constant matrix $\mathbb{C}$ was defined by (1.10). We write $\mathcal{H}(t) \phi$ in the form

$$
\mathcal{H}(t) \phi=\sum_{1}^{4} \mathcal{H}_{l}(t) \phi
$$

where

$$
\begin{aligned}
\mathcal{H}_{l}(t) \vec{\phi} & =\sum_{j=1}^{M} \int_{0}^{t} \Psi_{l}(x, t-\tau) \phi_{j}(\tau) d \tau \\
\Psi_{1}(x, t) & =\frac{1}{2 \pi i} \frac{1}{2 \pi i} \int d \xi e^{\xi t} \int d p e^{p x} \frac{K(p)}{K(p)+\xi}\left(\frac{1}{p^{j}}-\sum_{j=1}^{M} \xi^{-1+\frac{N+j}{\alpha}} \frac{\widetilde{\theta}_{j}(\xi, y)}{p^{N+j}}\right), \\
14) & \\
\Psi_{2}(x, t) & =-\frac{1}{2 \pi i} \frac{1}{2 \pi i} t^{-1-\frac{1}{\alpha}(1-j)} \int d \xi e^{\xi} \int d p e^{p x t^{-\frac{1}{\alpha}}} \frac{Y^{+} K(p)}{K(p)+\xi} \widetilde{I}_{1}^{+}(p, \xi) \\
& =\frac{1}{2 \pi i} \frac{1}{2 \pi i} t^{-1-\frac{1}{\alpha}(1-j)} \int d \xi e^{\xi} \int d p e^{p x t^{-\frac{1}{\alpha}}} \frac{Y^{+} \xi}{K(p)+\xi} \widetilde{I}_{1}^{+}(p, \xi),
\end{aligned}
$$

where

$$
\begin{gathered}
\widetilde{I}_{1}(z, \xi, y)=\frac{1}{2 \pi i} \int_{-i \infty}^{i \infty} \frac{d q}{q-z} \frac{1}{Y^{+}} \frac{K_{1}(q)-K(q)}{K_{1}(q)+\xi} \frac{1}{q^{j}} \\
\Psi_{3}(x, t)=\frac{1}{2 \pi i} \frac{1}{2 \pi i} \int_{-i \infty}^{i \infty} d \xi e^{\xi t} \int_{-i \infty}^{i \infty} d p e^{p x} \frac{Y^{+} \xi}{K(p)+\xi} \widetilde{I}_{2}^{+}(p, \xi, y)
\end{gathered}
$$


where

$$
\widetilde{I}_{2}(z, \xi, y)=-\frac{1}{2 \pi i} \int_{-i \infty}^{i \infty} \frac{d q}{q-z} \frac{1}{Y^{+}} \frac{K_{1}(q)-K(q)}{K_{1}(q)+\xi} \sum_{j=1}^{M} \xi^{\frac{N+j}{\alpha}} \frac{\widetilde{\theta}_{j}(\xi)}{q^{N+j}} d q
$$

A similar consideration to that in the book [4] proves that for $n=0,1, \ldots,[\alpha]$,

$$
\left\|\partial_{x}^{n} \mathcal{H}_{l}(t) \vec{h}\right\|_{\mathbf{L}^{s}} \leq C t^{-\frac{1}{\alpha}\left(\frac{1}{r}-\frac{1}{s}\right)-\frac{n}{\alpha}}\|\vec{h}(\cdot)\|_{\mathbf{L}^{r}} .
$$

Now we get an estimate for $\mathcal{H}_{2}(t) \vec{h}$. Using the estimate

$$
\left|\widetilde{I}_{1}^{+}(p, \xi, y)\right| \leq C \int_{\mathcal{C}_{2}} \frac{d q}{|q-p|} \frac{|q|^{\alpha-\mu-j}}{\left|K_{1}(q)+\xi\right|}
$$

from the first representation of (4.14), we get for $n<\delta<\alpha+n$ and $x>1$

$$
\begin{aligned}
\left|\partial_{x}^{n} \mathcal{H}_{2}(t) \vec{h}\right| & \leq C \frac{1}{\langle x\rangle^{\delta}} \sum_{j=1}^{N} \int_{0}^{t}\left|h_{j}(\tau)\right|(t-\tau)^{-1-\frac{1}{\alpha}(1-j+n-\delta)} d \tau \\
& \times \int_{\mathcal{C}_{3}} e^{-C|\xi|}|\xi| d \xi \int_{\mathcal{C}_{1}} d p \frac{|p|^{\alpha+n-\delta}}{|K(p)+\xi|} \int_{\mathcal{C}_{2}} \frac{d q}{|q-p|} \frac{|q|^{\alpha-\mu-j}}{\left|K_{1}(q)+\xi\right|} \\
& \leq C \frac{1}{\langle x\rangle^{\delta}} \sum_{j=1}^{N} \int_{0}^{t}\left|h_{j}(\tau)\right|(t-\tau)^{-1+\gamma} d \tau \\
& \leq C \frac{1}{\langle x\rangle^{\delta}}\|\vec{h}(\cdot)\|_{L^{\infty}} .
\end{aligned}
$$

In the case $x<1$, we use the second relation of (4.14). After integrating by parts we get

$$
\begin{aligned}
\mathcal{H}_{2}(t) \vec{h} & =\sum_{j=1}^{N} \int_{0}^{t} d \tau h_{j}^{\prime}(\tau)(t-\tau)^{-\frac{1}{\alpha}(1-j)} \\
& \times \int_{\mathcal{C}_{3}} d \xi e^{\xi} \int_{\mathcal{C}_{1}} d p e^{p x(t-\tau)^{-\frac{1}{\alpha}}} \frac{Y^{+}}{K(p)+\xi} \widetilde{I}_{1}^{+}(p, \xi) \\
& +\sum_{j=1}^{N} h_{j}(t) \int_{\mathcal{C}_{3}} d \xi e^{\xi} \int_{\mathcal{C}_{1}} d p \frac{Y^{+}}{K(p)+\xi} \widetilde{I}_{1}^{+}(p, \xi) \\
& +\sum_{j=1}^{N} h_{j}(0) \int_{\mathcal{C}_{3}} d \xi e^{\xi} \int_{\mathcal{C}_{1}} d p e^{p x t^{-\frac{1}{\alpha}}} \frac{Y^{+}}{K(p)+\xi} \widetilde{I}_{1}^{+}(p, \xi) \\
& \leq C\left(\left\|h_{j}^{\prime}(\tau)\right\|_{L^{\infty}}+\|\vec{h}(\cdot)\|_{L^{\infty}}\right) t^{-\frac{N-n}{\alpha}}
\end{aligned}
$$


Therefore,

$$
\begin{aligned}
\left\|\partial_{x}^{n} \mathcal{H}_{2}(t) \vec{h}\right\|_{\mathbf{L}^{s}} & \leq \sum_{j=n+1}^{M} \int_{0}^{t} d \tau\left|h_{j}(\tau)\right|(t-\tau)^{-1-\frac{1}{\alpha}\left(1-j+n-\frac{1}{s}\right)} \\
& \times \int_{\mathcal{C}_{3}} e^{-C|\xi|} d \xi \int_{\mathcal{C}_{1}} d p \frac{|p|^{n-\frac{1}{s}}}{|K(p)+\xi|} \int_{\mathcal{C}_{2}} \frac{d q}{|q-p|} \frac{|q|^{\alpha-\mu-j}}{\left|K_{1}(q)+\xi\right|} \\
& \leq t^{-\frac{N-n}{\alpha}} \sum_{j=1}^{M}\left\|h_{j}(\cdot)\right\|_{L^{\infty}} \\
& \times \int_{\mathcal{C}_{3}} d \xi \int_{\mathcal{C}_{1}} d p \frac{|p|^{n-\frac{1}{s}}}{|K(p)+\xi|} \int_{\mathcal{C}_{2}} \frac{d q}{|q-p|} \frac{\{q\}^{-\mu}|q|^{\alpha-j}}{\left|K_{1}(q)+\xi\right|} \\
& \leq C\left(\left\|h_{j}^{\prime}(\tau)\right\|_{L^{\infty}}+\|\vec{h}(\cdot)\|_{L^{\infty}}\right) t^{-\frac{N-n}{\alpha}} .
\end{aligned}
$$

Since

$$
\left|\widetilde{I}_{2}^{+}(p, \xi, y)\right| \leq C \int_{C_{2}} \frac{d q}{|q-p|} \frac{|q|^{\alpha-\mu}}{\left|K_{1}(q)+\xi\right|} \sum_{j=1}^{M}\left|\frac{\xi}{q}\right|^{\frac{N+j}{\alpha}}\left|\widetilde{\theta}_{j}(\xi, y)\right|
$$

and $\left|\widetilde{\theta}_{j}(\xi, y)\right| \leq C|\xi|^{-\frac{j}{\alpha}}$, in the same way we can obtain

$$
\left\|\partial_{x}^{n} \mathcal{H}_{3}(t) \vec{h}\right\|_{\mathbf{L}^{s}} \leq C\left(\left\|\vec{h}_{j}^{\prime}(\cdot)\right\|_{L^{\infty}}+\|\vec{h}(\cdot)\|_{L^{\infty}}\right) t^{-\frac{N-n}{\alpha}}
$$

Thus, the second part of estimate (4.1) of Lemma 4 is proved.

Lemma 5. The function $G(x, y, t)$ defined by formula (1.5) is the Green function of problem (1.1), i.e., for all $y>0$,

$$
\left\{\begin{array}{c}
\left(\partial_{t}+\left|\partial_{x}\right|^{\alpha}\right) G(x, y, t)=0 \\
G(x, y, 0)=\delta(x-y) \\
\partial_{x}^{j-1} G(x, y, t)=0, j=1,2, \ldots,[\alpha]-\left[\frac{\alpha+1}{2}\right]+1 .
\end{array}\right.
$$

Proof. Taking the Laplace transforms with respect to $x$ and $t$ of $G(x, y, t)$, we get $(4.21)$

$$
\widehat{\widehat{G}}(p, y, \xi)=\frac{1}{K(p)+\xi}\left(e^{-p y}+K(p) \sum_{j=1}^{M} \xi^{-1+\frac{N+j}{\alpha}} \frac{\theta_{j}(\xi, y)}{p^{N+j}}-Y^{+} I^{+}(p, \xi, y)\right) .
$$

With the help of the integral representations (1.7) and (1.6), for sectionally analytic functions $Y(z, \xi)$ and $I(z, \xi)$, making use of the Sokhotzki-Plemelj formula (2.1) and relation (3.18), we can express

$$
\begin{aligned}
\widehat{\widehat{G}}(p, y, \xi)= & \frac{1}{K_{1}(p)+\xi} \\
& \left(e^{-p y}+K_{1}(p) \sum_{j=1}^{M} \xi^{-1+\frac{N+j}{\alpha}} \frac{\theta_{j}(\xi, y)}{p^{N+j}}-Y^{-} \prod_{j=1}^{M} \frac{\left(p-k_{j}\right)}{\left(p+k_{j}\right)} I^{-}(p, \xi, y)\right) .
\end{aligned}
$$

By definition (1.9)

$$
e^{-k_{l}(\xi) y}-\sum_{j=1}^{M} \xi^{\frac{N+j}{\alpha}} \frac{\theta_{j}(\xi, y)}{k_{l}^{N+j}}=0, l=1, \ldots, M,
$$


where $K_{1}\left(k_{l}\right)=-\xi, \operatorname{Re} k_{l}(\xi)>0$ for $\operatorname{Re} \xi>0$. Therefore $\widehat{\widehat{G}}(p, y, \xi)$ is an analytic function for $\operatorname{Re} p>0$ and, as a consequence,

$$
\begin{aligned}
\widehat{\widehat{G}}(p, y, \xi) & =\mathbb{P}^{-} \widehat{\widehat{G}}(p, y, \xi) \\
& =-\frac{1}{2 \pi i} \lim _{z \rightarrow p, \operatorname{Re} z>0} \int_{-i \infty}^{i \infty} \frac{1}{p-z} \widehat{\widehat{G}}(p, y, \xi) d p .
\end{aligned}
$$

We can rewrite relation (4.21) in the form

$$
\begin{aligned}
& \xi \widehat{\widehat{G}}(p, y, \xi)-e^{-p y}+K(p)\left(\widehat{\widehat{G}}(p, y, \xi)-\xi^{-1+\frac{N+j}{\alpha}} \sum_{j=1}^{M} \frac{\theta_{j}(\xi, y)}{p^{N+j}}\right) \\
& =-Y^{+}(p, \xi) I^{+}(p, \xi, y) .
\end{aligned}
$$

Taking operator $\mathbb{P}^{-}$to the last relation and using (4.22) we obtain,

$$
\begin{aligned}
\xi \widehat{\widehat{G}}(p, y, \xi) & -e^{-p y}+\mathbb{P}^{-}\left\{K(p)\left(\widehat{\widehat{G}}(p, y, \xi)-\xi^{-1+\frac{N+j}{\alpha}} \sum_{j=1}^{M} \frac{\theta_{j}(\xi, y)}{p^{N+j}}\right)\right\} \\
& =-\mathbb{P}^{-}\left\{Y^{+}(p, \xi) I^{+}(p, \xi, y)\right\} .
\end{aligned}
$$

Since $Y^{+}(p, \xi) I^{+}(p, \xi, y)$ is a left limiting value on an analytic function in $\operatorname{Re} p<0$, it is easy to see that

$$
\mathbb{P}^{-}\left\{Y^{+}(p, \xi) I^{+}(p, \xi, y)\right\}=0 .
$$

Thus we finally get

$$
\xi \widehat{\widehat{G}}(p, y, \xi)-e^{-p y}+\mathbb{P}^{-}\left\{K(p)\left(\widehat{\widehat{G}}(p, y, \xi)-\xi^{-1+\frac{N+j}{\alpha}} \sum_{j=1}^{M} \frac{\theta_{j}(\xi, y)}{p^{N+j}}\right)\right\}=0 .
$$

Since all integrals of formula (1.5) converge absolutely by the Fubini theorem and the Cauchy theorem, we have

$$
\left.\widehat{G}(p, y, t)\right|_{t=0}=e^{-p y} .
$$

Also from (4.21) we have

$$
\widehat{\widehat{G}}(p, y, \xi)=\xi^{-1+\frac{N+j}{\alpha}} \sum_{j=1}^{M} \frac{\theta_{j}(\xi, y)}{p^{N+j}}+O\left(p^{-\alpha-1}\right),
$$

and therefore

$$
\left.\partial_{x}^{j-1} \widehat{G}(x, y, \xi)\right|_{x=0}=\left\{\begin{array}{c}
0, j=0, \ldots, N \\
\xi^{-1+\frac{N+j}{\alpha}} \theta_{j}(\xi, y), j=N+1, \ldots, N+M=[\alpha]+1 .
\end{array}\right.
$$

By the definition of operator $\left|\partial_{x}\right|^{\alpha}$, applying inverse Laplace transform to relation (4.23), and using (4.24) and (4.25), we get

$$
\left\{\begin{array}{c}
\left(\partial_{t}+\left|\partial_{x}\right|^{\alpha}\right) G(x, y, t)=0 \\
G(x, y, 0)=\delta(x-y) \\
\partial_{x}^{j-1} G(x, y, t)=0, j=1,2, \ldots,[\alpha]-\left[\frac{\alpha+1}{2}\right]+1
\end{array}\right.
$$

The lemma is proved. 


\section{Proof of Theorem 1}

From Lemma 5 by the Duhamel theorem, we get that the solution of the problem (1.1) with $h_{j}(t)=0, j=1, \ldots, N$ takes the form

$$
u(x, t)=\mathbb{G} u_{0}+\int_{0}^{t} \mathbb{G}(t-t) f(\tau) d \tau .
$$

Therefore taking in the problem (1.1) new function

$$
v(x, t)=u(x, t)-\sum_{j=1}^{N} \frac{x^{j-1} h_{j}(t)}{(j-1) !}
$$

we get for the solution $u(x, t)$ of problem (1.1)

$$
\begin{aligned}
u(x, t) & =\mathbb{G}\left(u_{0}-\sum_{j=1}^{N} \frac{x^{j-1} h_{j}(0)}{(j-1) !}\right)+\sum_{j=1}^{N} \frac{x^{j-1} h_{j}(t)}{(j-1) !} \\
& +\int_{0}^{t} \mathbb{G}(t-t)\left(f(\tau)-\left(\partial_{\tau}+\left|\partial_{x}\right|^{\alpha}\right) \sum_{j=1}^{N} \frac{x^{j-1} h_{j}(\tau)}{(j-1) !}\right) d \tau .
\end{aligned}
$$

Integrating by parts the last relation and using $\left|\partial_{x}\right|^{\alpha} \sum_{j=1}^{N} \frac{x^{j-1} h_{j}(t)}{(j-1) !}=0$, we find

$$
u(x, t)=\mathbb{G} u_{0}+\int_{0}^{t} \mathbb{G}(t-t) f(\tau) d \tau-\int_{0}^{t} \mathbb{G}_{t}(t-t) \sum_{j=1}^{N} \frac{x^{j-1} h_{j}(\tau)}{(j-1) !} d \tau .
$$

Since $\left(\partial_{t}+\left|\partial_{x}\right|^{\alpha}\right) G=0$, we get

$$
\begin{aligned}
& \int_{0}^{t} G_{t}(t-t) \sum_{j=1}^{N} \frac{x^{j-1} h_{j}(t)}{(j-1) !} d \tau \\
& =-\sum_{j=1}^{N} \frac{1}{(j-1) !} \int_{0}^{t} d \tau h_{j}(\tau) \int_{0}^{+\infty}\left|\partial_{x}\right|^{\alpha} G(x, y, t-\tau) y^{j-1} d \tau \\
& =-\sum_{j=1}^{N} \frac{(-1)^{j-1}}{(j-1) !} \int_{0}^{t} d \tau h_{j}(\tau) \lim _{p \rightarrow 0} \partial_{p}^{j-1} \int_{0}^{+\infty} e^{-p y}\left|\partial_{x}\right|^{\alpha} G(x, y, t-\tau) d \tau \\
& \left.=-\sum_{j=1}^{N} \frac{(-1)^{j-1}}{(j-1) !} \int_{0}^{t} d \tau h_{j}(\tau) \lim _{p \rightarrow 0} \partial_{p}^{j-1}\left|\partial_{x}\right|^{\alpha} \widehat{G(x, p}, t-\tau\right) .
\end{aligned}
$$

Therefore solution $u(x, t)$ of problem (1.1) has the form

$$
\begin{aligned}
u(x, t) & =\int_{0}^{+\infty} G(x, y, t) u_{0}(y) d y+\int_{0}^{t} \int_{0}^{+\infty} G(x, y, t-\tau) f(y, \tau) d y d \tau \\
& \left.+\sum_{j=1}^{N} \frac{(-1)^{j-1}}{(j-1) !} \int_{0}^{t} h_{j}(\tau) \lim _{p \rightarrow 0} \partial_{p}^{j-1}\left|\partial_{x}^{\alpha}\right| \widehat{G(x, p, t}-\tau\right) d \tau .
\end{aligned}
$$

From Lemma 4 we get $u(x, t) \in \mathbf{C}^{0}\left([0, T], \mathbf{H}_{2}^{N} \cap \mathbf{C}^{N}\right) \cap \mathbf{C}^{0}\left((0, T], \mathbf{H}_{2}^{[\alpha]} \cap \mathbf{C}^{[\alpha]}\right)$. From Theorem 3 this solution is unique. Theorem 1 is proved. 


\section{REFERENCES}

[1] Dong, Jianping; Xu, Mingyu. Applications of continuity and discontinuity of a fractional derivative of the wave functions to fractional quantum mechanics. J. Math. Phys. 49 (2008), no. 5, 052105, 16 pp. MR2421898 (2009h:81088)

[2] Galucio, A. C.; Deü, J.-F.; Dubois, F. The $G^{\alpha}$-scheme for approximation of fractional derivatives: application to the dynamics of dissipative systems. J. Vib. Control 14 (2008), no. 9-10, 1597-1605. MR2462967 (2009k:65111)

[3] Hayashi, N.; Kaikina, E. I.; Naumkin, P. I.; Shishmarev, I. A. Asymptotics for dissipative nonlinear equations. Lecture Notes in Mathematics, 1884. Springer-Verlag, Berlin, 2006, 557 pp. MR2230524 (2009d:35001)

[4] Hayashi, Nakao; Kaikina, Elena. Nonlinear theory of pseudodifferential equations on a halfline. North-Holland Mathematics Studies, 194. Elsevier Science B.V., Amsterdam, 2004, 319 pp. MR2031425 (2005b:35309)

[5] Kaikina, Elena I. Subcritical pseudodifferential equation on a half-line with nonanalytic symbol. Differential Integral Equations 18 (2005), no. 12, 1341-1370. MR.2174976 (2006f:35311)

[6] Kaikina, Elena I. Pseudodifferential operator with a nonanalytic symbol on a half-line, J. of Mathematical Physics 48, (2007), no. 11, 1341-1370. MR2370252(2008k:35521)

[7] Kilbas, A. A.; Srivastava, Hari M.; Trujillo, Juan J. Theory and applications of fractional differential equations. North-Holland Mathematics Studies, 204. Elsevier Science B.V., Amsterdam, 2006. MR2218073 (2007a:34002)

[8] Lakshmikantham, V.; Leela, S.; Vasundhara, Devi. Theory of Fractional Dynamic Systems, Cambridge Academic Publishers, Cambridge, 2009

[9] Naumkin, P. I.; Shishmarev, I. A. Nonlinear Nonlocal Equations in the Theory of Waves, Translations of Monographs, 133, A.M.S., Providence, R.I., 1994. MR.1261868 (94m:35230)

[10] Ortigueira, Manuel Duarte; Batista, Arnaldo Guimarães. On the relation between the fractional Brownian motion and the fractional derivatives. Phys. Lett. A 372 (2008), no. 7, 958-968. MR2393587 (2009b:60121)

[11] Ostrovsky, L. A. Short-wave asymptotics for weak-shock waves and solitons in mechanics, Int. J. Non-Linear Mechanics, 11 (1976), pp. 401-416. MR0443516 (56:1885)

[12] Ott, E.; Sudan, R. N. Nonlinear theory of ion acoustic waves with Landau damping, Phys. Fluids, 12, no. 11 (1969), pp. 2388-2394. MR0258349 (41:2996)

[13] Rossikhin, Yu. A.; Shitikova, M. V. Fractional-derivative viscoelastic model of the shock interaction of a rigid body with a plate. J. Engrg. Math. 60 (2008), no. 1, 101-113. MR 2374232 (2008j:74011)

[14] Samko, S. G.; Kilbas, A. A.; Marichev, O. I. Fractional Integrals and Derivatives. Theory and Applications, Gordon and Breach, Yverdon, 1993. MR1347689 (96d:26012)

[15] Silva, Manuel F.; Tenreiro Machado, J. A.; Barbosa, Ramiro S. Using fractional derivatives in joint control of hexapod robots. J. Vib. Control 14 (2008), no. 9-10, 1473-1485. MR2463073

[16] Tarasov, Vasily E. Differential equations with fractional derivative and universal map with memory. J. Phys. A 42 (2009), no. 46, 465102, 13 pp. 37. MR2552008

Instituto de Matemáticas, unam Campus Morelia, AP 61-3 (Xangari), Morelia CP 58089, MichoaCÁn, MEXiCO

E-mail address: ekaikina@matmor.unam.mx 